\title{
Percutaneous Endoscopic Necrosectomy (PEN): Is the PEN Mightier Than the VARD?
}

\author{
Guru Trikudanathan ${ }^{1}$ \\ Published online: 13 August 2019 \\ (c) Springer Science+Business Media, LLC, part of Springer Nature 2019
}

Necrotizing pancreatitis, one of the most feared and morbid complications of acute pancreatitis (AP), is seen in $5-10 \%$ of AP patients [1]. Very often, necrotic collections get infected which warrants drainage and debridement. Infected necrosis, typically a late event in the natural course of AP, can occur early in a quarter of the patients [2]. Typically, this is managed conservatively with intravenous antibiotics as current guidelines recommend delaying invasive intervention for at least 4 weeks after initial presentation to allow the collection to become "walled off" [3]. Occasionally necrotizing pancreatitis with clear evidence of infection despite maximal medical support may warrant an earlier intervention for drainage and/or debridement [2].

The current paradigm for intervention in necrotizing pancreatitis involves a staged multi-disciplinary algorithmic step-up approach with endoscopic transluminal drainage (ETD) or percutaneous drainage (PCD) as the initial step based on the location of the necrotic collection and the availability of localized expertise $[1,3]$. PCD is widely available and is the initial drainage procedure of choice, especially for necrotic collections that may not be readily amenable to ETD such as those that do not abut the lumen of the stomach and duodenum or that extend deeply into the retroperitoneum $[4,5]$. In PCD, drainage of infected fluid under pressure temporizes septic complications, which further accelerates clinical improvement and enabling further encapsulation of necrotic collections [4]. Freeny et al. [5] reported complete resolution of sepsis in $47 \%$ of patients with infected necrosis after aggressive percutaneous drainage that required an average of four catheter insertions and lavage every $8 \mathrm{~h}$ for a mean of 85 days. In the landmark PANTER study, 35\% of patients with infected necrosis recovered with antibiotic

Guru Trikudanathan

triku001@umn.edu

1 Division of Gastroenterology, Hepatology and Nutrition, University of Minnesota, 406 Harvard St SE, MMC36, Minneapolis, MN 55455, USA treatment and PCD as the sole therapeutic modality, with the caveat that solid necrotic tissue cannot be effectively evacuated via small caliber catheters. Large areas of necrosis, poor liquefaction, diffuse and multifocal collections, and the presence of multi-organ failure are factors that predict a poor response to $\mathrm{PCD}[6,7]$ combined with the observation that when PCD was used alone, adverse events such as pancreatico-cutaneous fistulae were reported in up to $27 \%$ of patients [1]. Dual-modality drainage with concurrent endoscopic transluminal and percutaneous drainage was conceived in order to reduce rates of pancreatic and enteric fistulae [8]. While lavage through the percutaneous catheter with egress through the transmural fistula facilitates removal of liquefied necrotic material, solid necrotic material often requires direct debridement for complete resolution.

Various forms of minimally invasive retroperitoneal necrosectomy techniques, such as video-assisted retroperitoneal debridement (VARD), and debridement using a nephroscope or mediastinoscope have been described [9]. VARD, which requires a 5-7-cm flank incision for insertion of a laparoscope, an irrigation catheter, and open surgical forceps, was recently compared to an endoscopic transluminal approach in a randomized trial (TENSION study) and had increased rates of pancreatic fistulae and increased lengths of stay [7]. All of the above instruments (laparoscope, nephroscope, and mediastinoscope) are rigid, limiting maneuverability around vital structures and penetration into deep recesses.

Percutaneous endoscopic necrosectomy (often referred to as sinus tract endoscopy) using flexible endoscopy was pioneered as a technique which utilizes the existing percutaneous tract to facilitate retroperitoneal necrosectomy using instrumentation established for endoscopic transluminal necrosectomy [10]. The angulation and versatility offered by the flexible endoscope make it particularly convenient to access various extensions deep within the retroperitoneum. Although when initially described, this procedure was performed under general anesthesia, Dhingra et al. [11] 
demonstrated its safety and feasibility when performed under moderate conscious sedation at bedside in a pilot study.

In this issue of Digestive Diseases and Sciences, the same group reported a larger follow-on study conducted prospectively in 272 necrotizing pancreatitis patients of whom 177 (65\%) developed infected necrosis [notably 142 (59\%) with acute necrotic collection] [12]. Since the majority of the studies of necrotizing pancreatitis use selected patients felt suitable for a particular technique or intervention, this group should be lauded for studying an unselected cohort. Among these 177 patients with infected necrosis, 102 (72\%) patients had an infected acute necrotic/fluid collection and 75 (42\%) had infected walled-off necrosis. Roughly one-third underwent PEN, and 30\% needed rescue open necrosectomy following PEN.

Among the 102 with infected acute necrotic/fluid collection, 20 were managed with antibiotics alone, 82 underwent PCD, 26 underwent PEN, and 27 required open necrosectomy. A $44 \%$ mortality was reported in the group with infected acute necrotic/fluid collection due to persistent organ failure. Among the 75 patients with infected walledoff necrosis, seven were managed with antibiotics alone, 19 patients managed on PCD alone, 27 underwent PEN, and 19 underwent open necrosectomy. A $28 \%$ mortality of was reported from infected walled-off necrosis. A $64 \%$ overall successful resolution after PEN was reported. On the basis of these data, the authors concluded that independent predictors of mortality included extensive necrosis and early organ failure.

Organ failure is one of the key determinants of severity and mortality associated with acute pancreatitis [1]. It is typically biphasic with an early (primary) organ failure typically driven by intense systemic perturbation from a sterile inflammation in contrast to late (secondary) organ failure due to sepsis from infected necrosis. In the present study, the majority of the patients were critically ill with $170(78 \%)$ with early organ failure and more than half with multi-organ failure where the prognosis is typically guarded. Not surprisingly, reported mortality was substantial (38\%) with the majority likely to have succumbed to early organ failure further supported by the increased APACHE II and Marshall scores on admission seen among the non-survivors. No procedure-related mortality was reported in this study.

Our group recently explored the role of an early ( $<4$ weeks) endoscopically centered step-up approach in the face of clinical deterioration with organ failure and demonstrated acceptable results without any difference in complications, compared with a standard intervention after 4 weeks. Among the 76 who underwent early interventions (ETD-62\% and PCD-29\%), more than half had only partial degree of encapsulation [2]. Both of these studies suggest that patients with infected necrosis and clinical deterioration need not necessarily wait for an arbitrary 4-week cut-off period. Only a multi-disciplinary team should perform any early intervention with pancreatic surgeons and an interventional radiology team available for back-up in the event of a complication. ETD is preferred for a nearly encapsulated collection that is closely adherent to the stomach and duodenum and where a safe retroperitoneal window is not available, whereas PCD is favored for poorly demarcated collection, septic unstable patients, or for extra-pancreatic collections distant from the stomach or duodenum.

This study demonstrated the safety and feasibility of performing PEN at the bedside, under moderate conscious sedation, obviating need for general anesthesia. General anesthesia is known to provoke systemic inflammation in these critically ill patients and is universally utilized for peroral endoscopic necrosectomy. Although not shown here, PEN is likely to be cost-effective and future studies should estimate its economic benefits. Nevertheless, as discussed in the study, PEN should only be attempted through a retroperitoneal approach as an anterior transperitoneal approach may result in enteral leaks and the dissemination of infected material with resultant peritonitis. The percutaneous tract needs to mature, before attempting PEN (an average of 17 days shown in this study). Risks of bleeding and persistent pancreatico-cutaneous fistulae are other considerations. PEN particularly when performed with a pediatric gastroscope is a labor-intensive procedure that needs a significant time commitment. Further research is imperative to develop dedicated accessories for PEN. Since it is unclear how long the drains were left in situ in this study, future guidelines should address drain management strategies after PEN.

The percutaneous approach demands a dedicated team of interventional radiologists willing to assiduously manage these patients, paying meticulous attention toward catheter care, frequent upsizing of catheters and using imaging to localize undrained areas. Percutaneous endoscopic necrosectomy appears promising as a sequential step-up strategy in appropriately selected patients, when used in a tertiary care setting serving to expand the existing armamentarium for management of large necrotic collections with deep retroperitoneal extension.

\section{References}

1. Trikudanathan G, Wolbrink DRJ, van Santvoort HC, Mallery S, Freeman M, Besselink MG. Current concepts in severe acute and necrotizing pancreatitis: an evidence-based approach. Gastroenterology. 2019;156:1994-2007.

2. Trikudanathan G, Tawfik P, Amateau SK, et al. Early ( $<4$ weeks) versus standard ( $\geq 4$ weeks) endoscopically centered step-up interventions for necrotizing pancreatitis. Am J Gastroenterol. 2018;113:1550-1558.

3. Arvanitakis M, Dumonceau J-M, Albert J, et al. Endoscopic management of acute necrotizing pancreatitis: European Society of 
Gastrointestinal Endoscopy (ESGE) evidence-based multidisciplinary guidelines. Endoscopy. 2018;50:524-546.

4. Trikudanathan G, Attam R, Arain MA, Mallery S, Freeman ML. Endoscopic interventions for necrotizing pancreatitis. Am J Gastroenterol. 2014;109:969-981. (quiz 982).

5. Freeny PC, Hauptmann E, Althaus SJ, Traverso LW, Sinanan M. Percutaneous CT-guided catheter drainage of infected acute necrotizing pancreatitis: techniques and results. AJR Am J Roentgenol. 1998;170:969-975.

6. van Santvoort HC, Besselink MG, Bakker OJ, et al. A step-up approach or open necrosectomy for necrotizing pancreatitis. $N$ Engl J Med. 2010;362:1491-1502.

7. van Brunschot S, van Grinsven J, van Santvoort HC, et al. Endoscopic or surgical step-up approach for infected necrotising pancreatitis: a multicentre randomised trial. Lancet. 2018;391:51-58.

8. Ross AS, Irani S, Gan SI, et al. Dual-modality drainage of infected and symptomatic walled-off pancreatic necrosis: long-term clinical outcomes. Gastrointest Endosc. 2014;79:929-935.

9. Horvath KD, Kao LS, Wherry KL, Pellegrini CA, Sinanan MN. A technique for laparoscopic-assisted percutaneous drainage of infected pancreatic necrosis and pancreatic abscess. Surg Endosc. 2001;15:1221-1225.
10. Carter CR, McKay CJ, Imrie CW. Percutaneous necrosectomy and sinus tract endoscopy in the management of infected pancreatic necrosis: an initial experience. Ann Surg. 2000;232:175-180.

11. Dhingra R, Srivastava S, Behra S, Vadiraj PK, Venuthurimilli A, et al. Single or multiport percutaneous endoscopic necrosectomy performed with the patient under conscious sedation is a safe and effective treatment for infected pancreatic necrosis (with video). Gastrointest Endosc. 2015;81:351-359.

12. Jain S, Padhan R, Bopanna S, Jain SK, Dhingra R, Dash NR, et al. Percutaneous endoscopic step-up therapy is an effective minimally invasive approach for infected necrotizing pancreatitis. Dig Dis Sci. (Epub ahead of print). https://doi.org/10.1007/s10620-01905696-2.

Publisher's Note Springer Nature remains neutral with regard to jurisdictional claims in published maps and institutional affiliations. 